\title{
Contractile Strength during Variable Heart Duration Is Species and Preload Dependent
}

\author{
Carlos A. A. Torres ${ }^{1,2}$ and Paul M. L. Janssen ${ }^{1}$ \\ ${ }^{1}$ Department of Physiology and Cell Biology, The Ohio State University, Columbus, OH 43210, USA \\ ${ }^{2}$ Department of Emergency Medicine, The Ohio State University, Columbus, OH 43210, USA
}

Correspondence should be addressed to Paul M. L. Janssen, janssen.10@osu.edu

Received 1 July 2011; Accepted 15 August 2011

Academic Editor: Henk Granzier

Copyright (C) 2011 C. A. A. Torres and P. M. L. Janssen. This is an open access article distributed under the Creative Commons Attribution License, which permits unrestricted use, distribution, and reproduction in any medium, provided the original work is properly cited.

\begin{abstract}
We investigate the effect of beat-to-beat variability on cardiac contractility. Cardiac trabeculae were isolated from the right ventricle of rabbits and beagle dogs and stimulated to isometrically contract, alternating between fixed steady state versus variable interbeat intervals. Trabeculae were stimulated at physiologically relevant frequencies for each species ( $\operatorname{dog} 1$ and $4 \mathrm{~Hz} ;$ rabbit 2 and $4 \mathrm{~Hz}$ ) intercalating fixed periods with $40 \%$ variability. A subset of the trabeculae (at $90 \%$ of optimal length) was stretched prior to stimulation between 5 and $13 \%$ and stimulated at the same frequencies with a fixed versus $40 \%$ variation. Fixed rate response at the same base frequency was measured before and after each variable period and the average force reported. In canine preparations no change in force was observed as a result of the imposed variability in beat-to-beat duration. In the rabbit, we observed a nonsignificant decrease in force between fixed and variable pacing at both 2 and $4 \mathrm{~Hz}(n=8)$ when $40 \%$ variability was introduced. When a $5 \%$ and $13 \%$ stretch was applied, the correlation coefficient sharply increased, indicating a more prominent impact of the prebeat duration on the following cycle with higher preload.
\end{abstract}

\section{Introduction}

Bowditch [1] described the influence of the interval between beats on force development over 100 years ago. His landmark experiments in frog hearts ascribed prime importance to the effect of the interval between beats on the strength of contraction. Widely recognized as one of the most important relationships in cardiac physiology, the force frequency relationship has ever since been subject of intense investigation in the field of cardiac mechanics. The force-frequency relationship (FFR) describes that an increase in force development is observed as the interval between beats decreases (higher frequency) and has been shown in vivo and in vitro, occurs in all healthy mammals [2], and this FFR is impaired in patients with heart failure [3]. Typically, these changes in contractile force develop over several beats and are generally derived from steady-state observations at different frequencies [4].

Other intrinsic and seemingly opposite phenomena in mammalian myocardium act on a more immediate nonsteady-state beat-to-beat basis. Postrest potentiation des- cribes the stronger force development seen when stimulation is resumed after a longer than usual interval, that is, a period or rest, between beats [5]. Mechanical restitution describes the dependency of the recovery of contractile force on the interval between contractions, namely, that a shorter interval leads to a weaker beat and a longer interval leads to a stronger beat $[6,7]$. Postextrasystolic potentiation is yet another mechanism whereby a shorter interval that precedes a longer interval determines a stronger contraction in the subsequent beat [8].

In our previous work in rat, we were able to show small gains in average contractile force over the fixed rate period given an equivalent number of beats in each period [9]. Here, to translate these findings to species that more closely resemble human cardiac function, we investigated $\mathrm{R}-\mathrm{R}$ interval modulation (such as what may occur during atrial fibrillation) may have on the inotropic status of the myocardium in rabbits and dogs and seek to verify the impact of this strategy on different species with different calcium handling and EC coupling properties. 


\section{Methods}

Male NZW rabbits (2-3 months) were anesthetized with pentobarbital (at $60 \mathrm{mg} / \mathrm{kg}$ intraperitoneal). In dogs (weighing $19.0 \pm 0.4 \mathrm{~kg}$, range, $13.6-24.1 \mathrm{~kg}, 2-3$ years old), an intravenous catheter was placed in the cephalic vein and a surgical plane of anesthesia was induced by the bolus injection (over 1-2 min) of sodium pentobarbital $(50 \mathrm{mg} / \mathrm{kg}$, Nembutal, Abbott, North Chicago, ILL). Under deep anesthesia, the hearts were rapidly removed and perfused retrogradely through the aorta with Krebs Henseleit solution $(120 \mathrm{mM}$ $\mathrm{NaCl}, 5 \mathrm{mM} \mathrm{KCl}, 2 \mathrm{mM} \mathrm{MgSO}_{4}, 1 \mathrm{mM} \mathrm{NaH} \mathrm{PO}_{4}, 20 \mathrm{mM}$ $\mathrm{NaHCO}_{3}, 0.25 \mathrm{mM} \mathrm{Ca}^{2+}$, and $10 \mathrm{mM}$ glucose) in equilibrium with $95 \% \mathrm{O}_{2} / 5 \% \mathrm{CO}_{2}$. 2,3-Butanedione monoxime (BDM) was added to the dissection solution to stop the heart from beating and to prevent damage during dissection [10]. This investigation conforms with the Guide for the Care and Use of Laboratory Animals published by the US national Institutes of Health (NIH Publication Ns 83-23, revised 1996). Suitable thin, uniform, nonbranched trabeculae along the free wall of the right ventricle were dissected carefully and without touching the muscle mounted in the bath as previously described for rabbit [11] and dog [12]. Average width was $\sim 120-140 \mu \mathrm{m}$ in order to prevent hypoxic cores of the muscles [13]. The dissected specimen contained small cubes of ventricular tissue attached to each end to facilitate mounting the muscle onto the experimental setup. The cube of ventricular tissue at one end of the trabecula was connected to a hook, and the cube at the other end rested in a basket-shaped extension of the force transducer. The muscle was bathed in a continuous flow of oxygenated K$\mathrm{H}$ solution (without $\mathrm{BDM}$ ), and with $1.75 \mathrm{mM} \mathrm{Ca}^{2+}$, at $37^{\circ} \mathrm{C}$. The dimensions (length, width, and thickness) of the muscle were measured under $30 \mathrm{x}$ magnification. Initially, the muscle was stimulated at $1 \mathrm{~Hz}$ at a temperature of $37^{\circ} \mathrm{C}$ and stretched until small increases in length resulted in about equal increases in resting tension and active developed tension. This fixed optimal preload is equivalent to that near the end diastolic volume in the in vivo situation (sarcomere length of $\sim 2.2 \mu \mathrm{m}$ ) [14]. Trabeculae were stimulated at physiologic frequencies for each species ( 1 and $4 \mathrm{~Hz}$ in dog, $n=9$, and 2 and $4 \mathrm{~Hz}$ in rabbit, $n=8$ ) with or without a $40 \%$ variation imposed on interbeat duration. In order to define the load dependence of contractility during heart rate variability (HRV), a subset of rabbit trabeculae was subsequently subjected to a variable preload protocol by utilizing a high-speed high-accuracy servomotor as a displacement device $(n=5)$. One end of the trabeculae was attached to a length displacement device (i.e., a high-speed, highaccuracy servomotor). Once peak tension is reached and forces start declining ( $150 \mathrm{~ms}$ after initiation of the twitch), the servomotor reduces the muscle length by $10 \%$ (to $90 \%$ of optimal) within $2 \mathrm{~ms}$, after which it is immediately stretched at the velocity of one muscle length per second until the next beat is triggered. At that point, the muscle length is kept constant. This range of lengths was chosen to be close to the in vivo sarcomere length range the heart undergoes during a contraction [14]. The protocol is then repeated (Figure 1). By actively stretching the muscle at a rate of 1 muscle length per second, a certain percent stretch of muscle length will result, which is identical to the percentage of muscle that is released. Thus, average preload is identical both during the fixed preload and variable preload protocol.

In all protocols, total number of beats in each fixed versus variable period was the same. A fixed-rate steady-state response at the same base frequency was measured before and after each variable period, and average force was reported.

Data Analysis. Data were obtained and analyzed through custom written software (LABVIEW). Correlation coefficients and slopes were obtained for prebeat duration and force development. Kaleidagraph statistical package was utilized to perform Students $t$-test and repeated measures ANOVA where appropriate. All data are reported as means \pm SEM.

\section{Results}

First, we compared the average active force development between protocols with base frequency with a random $40 \%$ variation in the interval between beats compared. In dog myocardium (Figure 2), there was no significant change on average force development at both $1 \mathrm{~Hz}$ (cycle time of $1000 \mathrm{~ms}$ ) and $4 \mathrm{~Hz}$ (cycle time of $250 \mathrm{~ms}$ ).

Next, we compared these protocols in the rabbit. Since during in vivo beats, a prolonged beat-interval increases filling time, we mimicked this increase an increase in preload by stretching the muscle during the interbeat period until stimulated. In Figure 3, we depict the observed nonsignificant decrease in strength when imposing a $40 \%$ variable protocol until a stretch (increased pre load) is applied. Albeit not significant, there is a reversal of the negative tendency at $5 \%$ stretch for both baseline frequencies 2 and $4 \mathrm{~Hz}$. Only at the more extreme $13 \%$ stretch at $4 \mathrm{~Hz}$ (the high end of the frequency range for rabbit) do we again observe a nonsignificant trend towards a negative effect.

In Figure 4, we show the correlation between prebeat duration and average force development in the dog at both 1 and $4 \mathrm{~Hz}$ during a $40 \%$ variable pacing protocol. Note the static relationship at the different frequencies. There is no difference with the increase in frequency.

In Figure 5, we show that as the preload stretch increases, while the baseline frequency increases too, a much higher correlation between beatduration and developed force. At $4 \mathrm{~Hz}$, the increase in correlation becomes statistically significant. The longer the prebeat duration, the higher the contractile force observed, and the shorter the interval, the weaker the following beat.

\section{Discussion}

In our previous work, we had suggested and shown that an algorithm that imposed a beat-to-beat variability can lead to an average slight increase in force in rat myocardium [9]. In subsequent studies, we showed that in the rabbit, up to three preceding beats contribute to the amplitude of a given beat but that the average amplitude is not significantly 


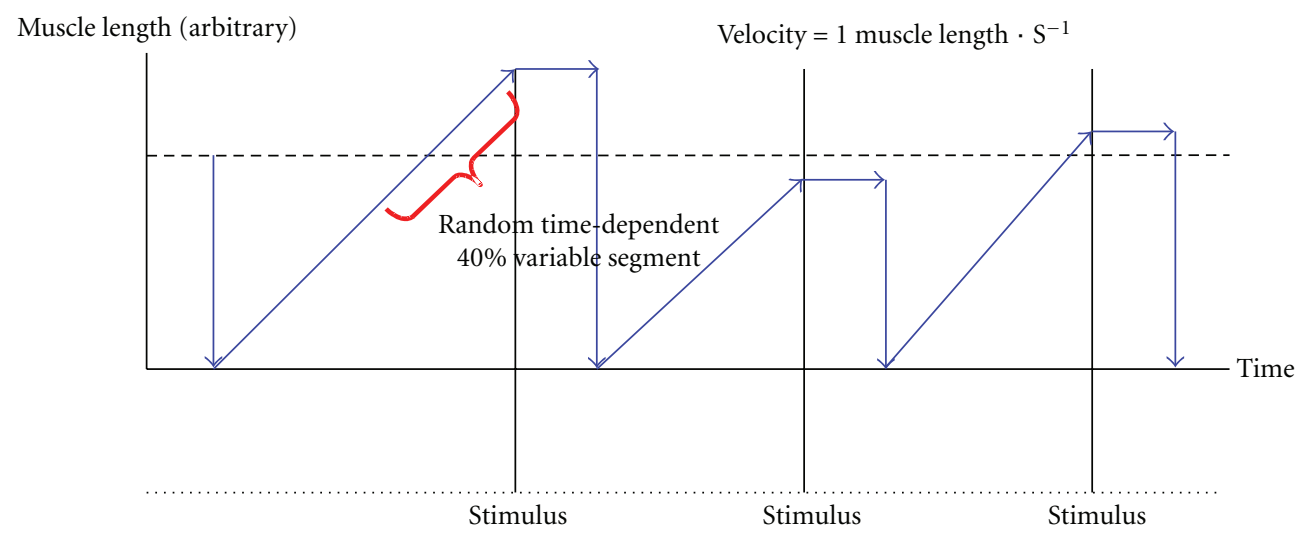

Figure 1: Following a stimulus, muscle length ( $y$-axis) is kept constant till well after peak active force development has been reached. At $150 \mathrm{~ms}$ after the stimulation, muscle length is quickly reduced to $90 \%$ of optimal length and thereafter restretched at 1 muscle length per second. The duration of this stretch varied resulting in successive beats with different preloads, where the preload is increased linearly with the interbeat duration.

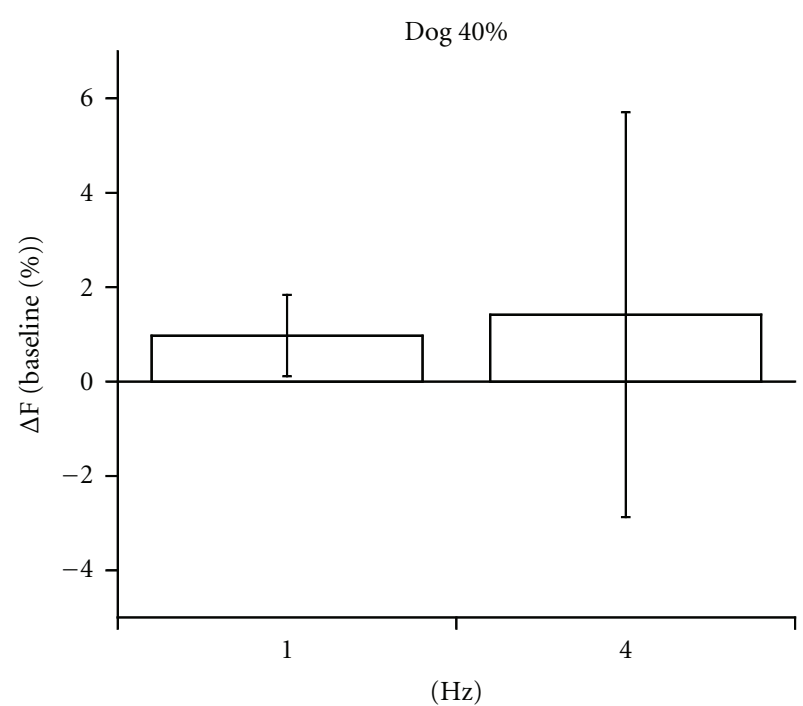

Figure 2: In dog trabeculae, at $40 \%$ variability, the average active developed force was not increased at 4 or $1 \mathrm{~Hz}$. Results are expressed compared to baseline.

affected by a pseudovariable heart rate compared to the average of that rate applied as steady state $[15,16]$. Here, we tested the efficacy of this initial randomized beat duration strategy in the rabbit and dog. The current data suggest that neither canine or rabbit myocardium seem to benefit (i.e., increase inotropy) from a variable rate with no negative or positive effect of variability on force development or kinetics noticed. The explanation for this species differences is more than likely found in the distinct manner in which EC coupling and calcium regulation are handled in each animal. Of particular interest is the increased contribution of the L-type calcium channels to the overall intracellular calcium pool during each contraction: $\sim 70 \%$ in rabbit and $\operatorname{dog}$ compared to less only $2-5 \%$ in rat $[17,18]$. In addition, it is known that also myofilament isoform composition differs between small and larger mammals, most notably in the

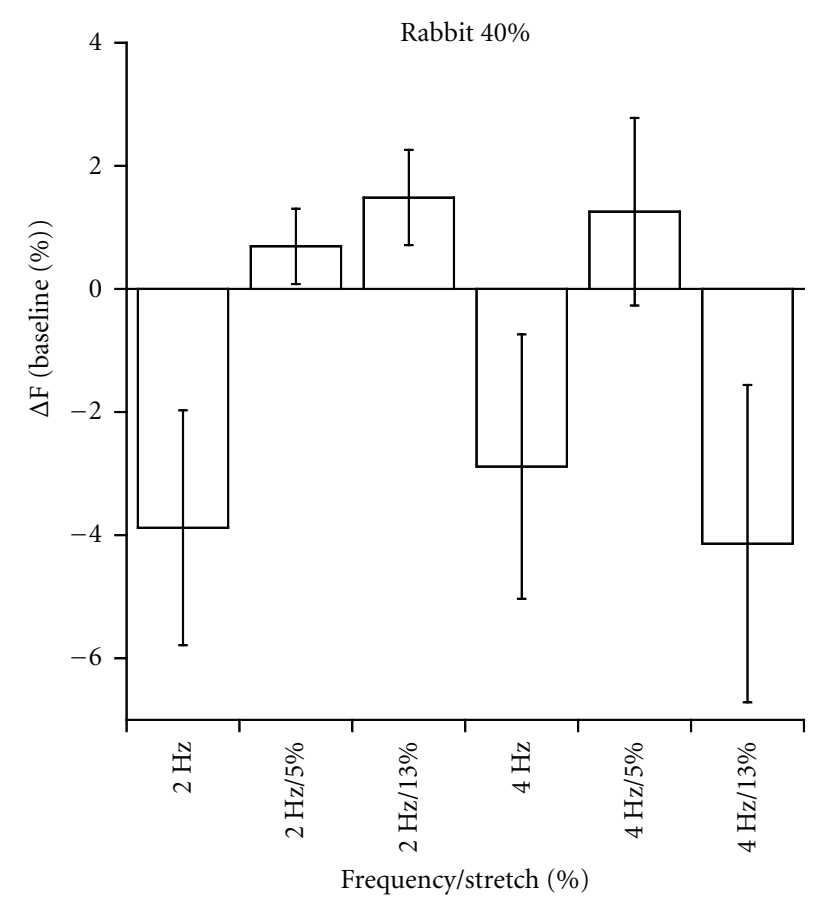

FIgure 3: At a variability of $40 \%$ in the rabbit at both 2 and $4 \mathrm{~Hz}$, there was no statistically significant difference between variable and base protocols. The addition of a $5 \%$ and/or $13 \%$ stretch just prior to contraction did not seem to improve or impair the average force development.

myosin heavy chain (MHC) isoform [19]. However, since the force of contraction highly depends on time passed since the last contraction, dynamic calcium handling and distribution of calcium fluxes that are very much time-dependent are thus more likely contributors, versus fixed differences in isoforms of MHC, or other differences in myofilament protein expression, although these cannot be excluded to contribute to the species differences at this point.

When next we investigated the effect of a variable length (load) under a variable pacing protocol on the rabbit heart, 


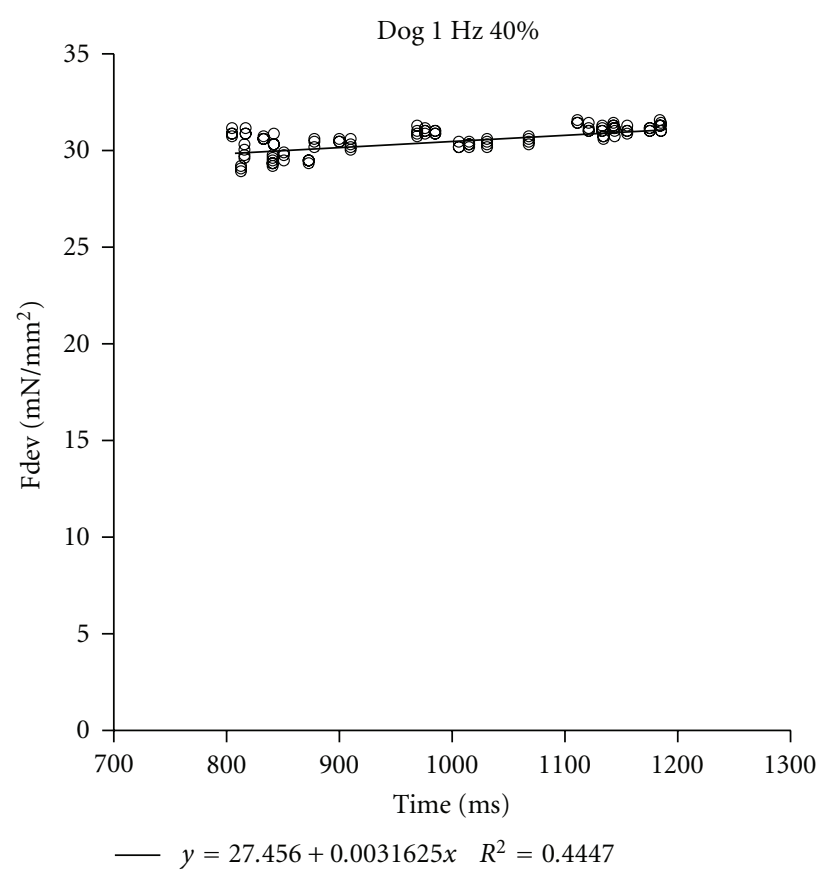

(a)

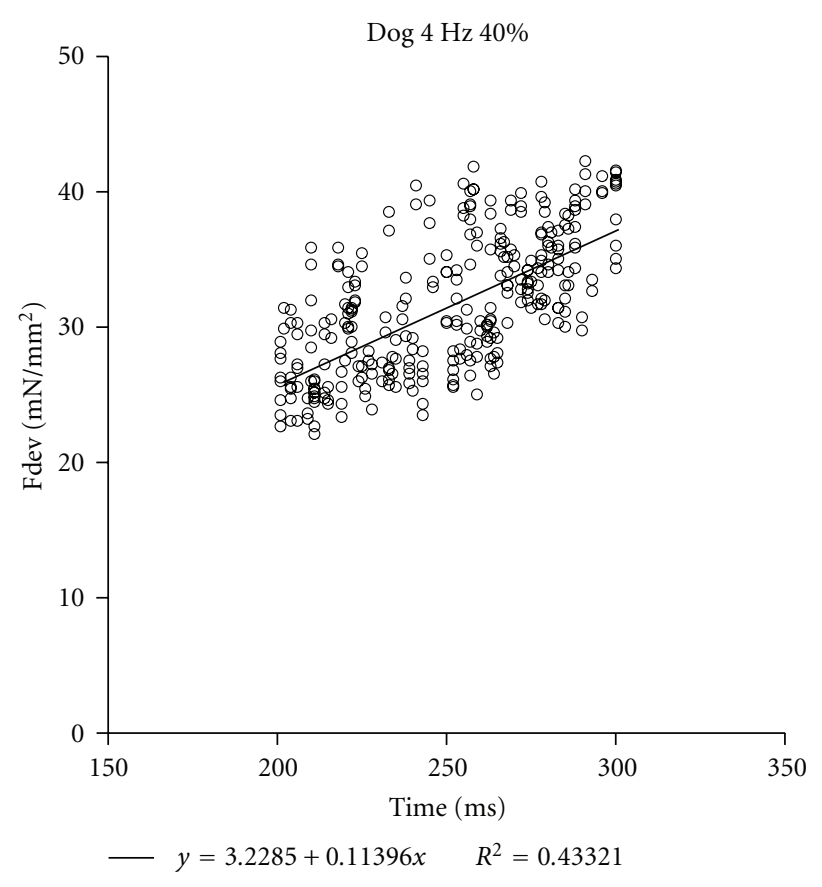

(b)

FIGURE 4: The correlation between prebeat duration and average force development in the dog is demonstrated for both 1 and $4 \mathrm{~Hz}$. There is no difference with the increase in frequency.

we noticed a discrete increase on average force of the variable over the fixed rate base frequency that was constant at both 2 and $4 \mathrm{~Hz}$ for the 5\% stretch group. At 13\% stretch, a similar effect was noticed at the $2 \mathrm{~Hz}$ frequency group, whereas, at $4 \mathrm{~Hz}$, there was a nonsignificant weakening effect. This latter finding may have resulted as these higher frequencies from the additional stretch may have caused some damage to the myofilament structure by a too rapid stretch.

Of greater importance was the significantly tighter correlation $R^{2}$ observed between the prebeat duration and the developed force once a small variable stretch was applied. This correlation was much tighter as the frequency and stretch increased $(P<0.001$ at $4 \mathrm{~Hz})$. This suggests that while the interval between beats is important, there is considerable effect that is load dependent affecting the subsequent beat. While several studies have shown an independent and strong effect of the interbeat duration alone and with contribution of the Frank Starling mechanism put into question [7, 20], there are others with findings similar to ours during in vivo investigations $[21,22]$. At the heart of the problem lie two seemingly opposite phenomena: on one hand a sustained decrease in interbeat duration (thus an increase in frequency) leads to increased contractility, whereas instantaneous decreases in interbeat duration lead to a weaker immediate first beat [23]. We here now show that this interplay appears to be nonlinear in the time domain and that, by manipulating the frequency and variability a host of factors interact dynamically in a non-steady-state of equilibrium that changes every instant. The mechanism of the stretchdependent "tightening" of the correlation is likely to be found in the interaction between load and calcium handling and could include stretch-dependent calcium channels and ion homeostasis [24] on one hand, an interaction of the higher calcium sensitivity at greater sarcomere length [25], or a greater contribution of predominantly passive elements such as collagen or titin [26]. Potentially, at larger sarcomere length, the increase in the absolute myofilament calcium buffer capacity is nonlinear with stretch and promotes retention of calcium between two subsequent beats. However, more detailed experiments would be required to test such hypothesis.

On a beat-to-beat basis with instantaneous switching intervals away from steady state, the phenomena of mechanical restitution and postrest potentiation and postextrasystolic potentiation likely play an important role in determining the immediate contractile state of the heart [27]. We speculate that the direction and the magnitude of force modulation possibly relate to the different relative contributions of the sodium calcium exchanger (NCX) versus the sarcoplasmic reticulum calcium ATPase (SERCA) to the contractile process in each species. This paper uniquely highlights the influence of heart rate variability on a beat-to-beat basis and the different effects in diverse species while attempting to account for real life in vivo events such as preload stretching of the fibers.

We conclude that, in larger mammals, no changes in contractile force can be obtained by introducing variation in the beat-to-beat duration, and we confirm the evidence that prebeat duration has an important effect on developed strength [15]. The addition of a prebeat stretch effectively tightens this association that also becomes more significant as the frequency increases. Short-term manipulation of the beat-to-beat 

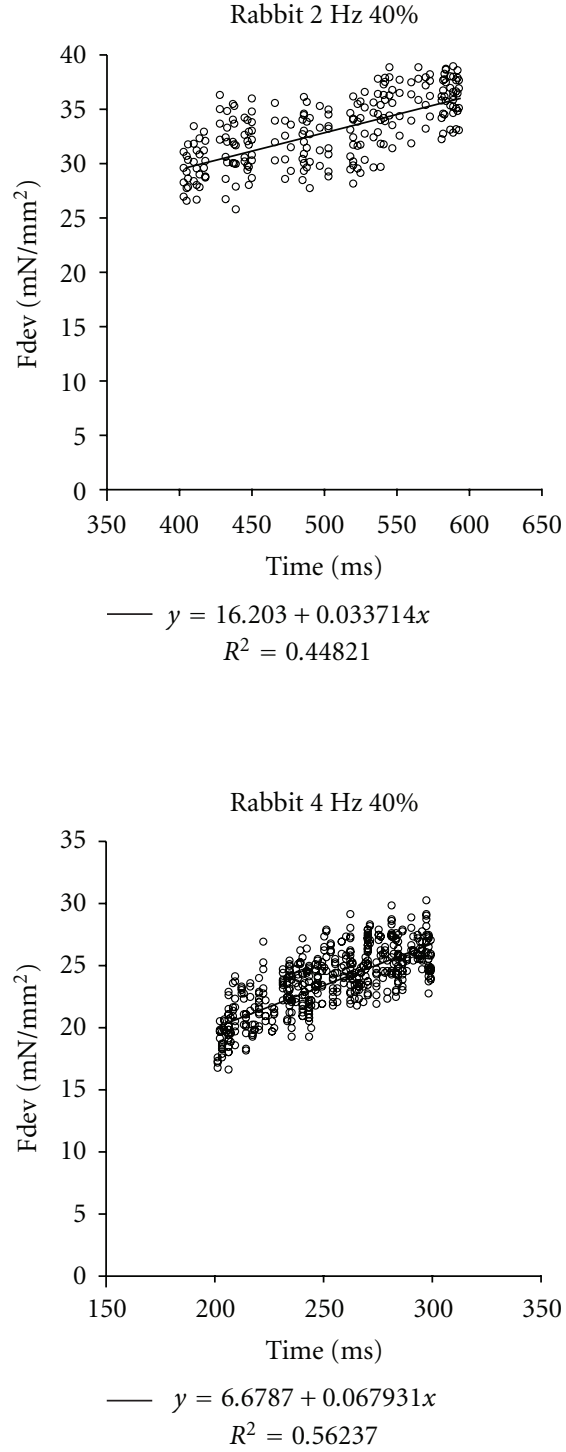

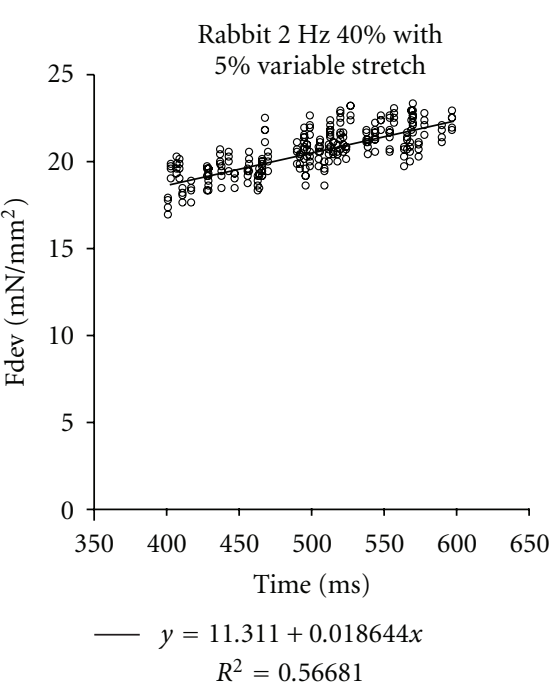

(a)
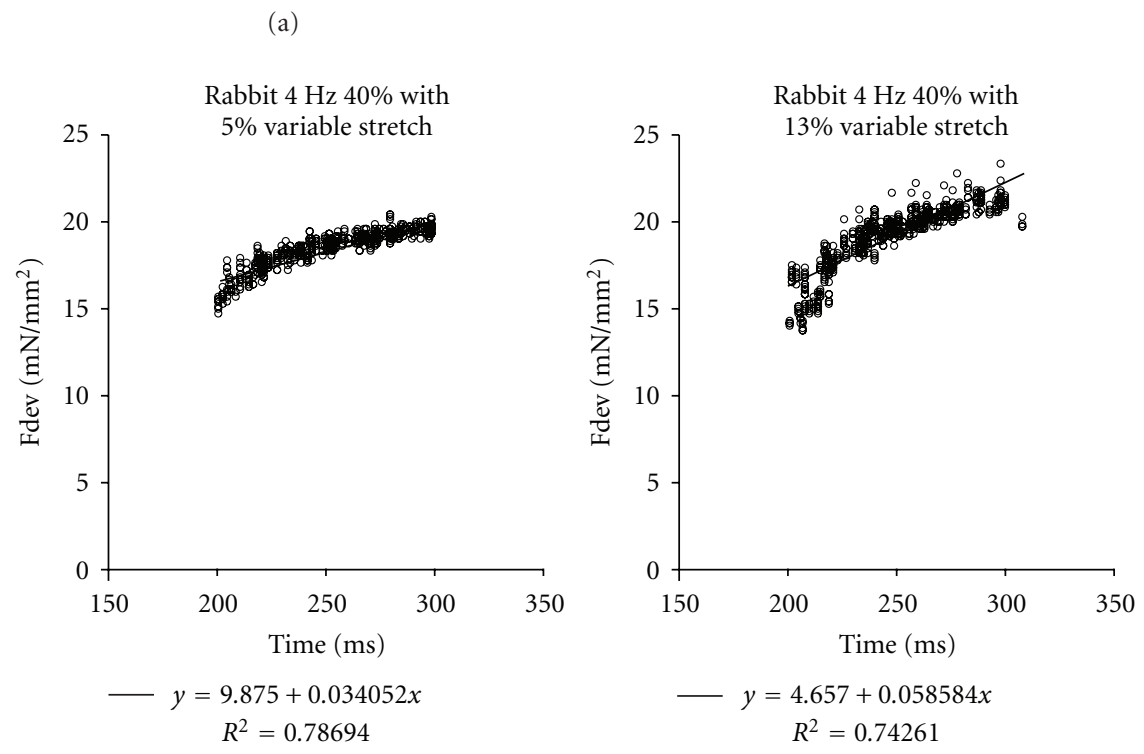

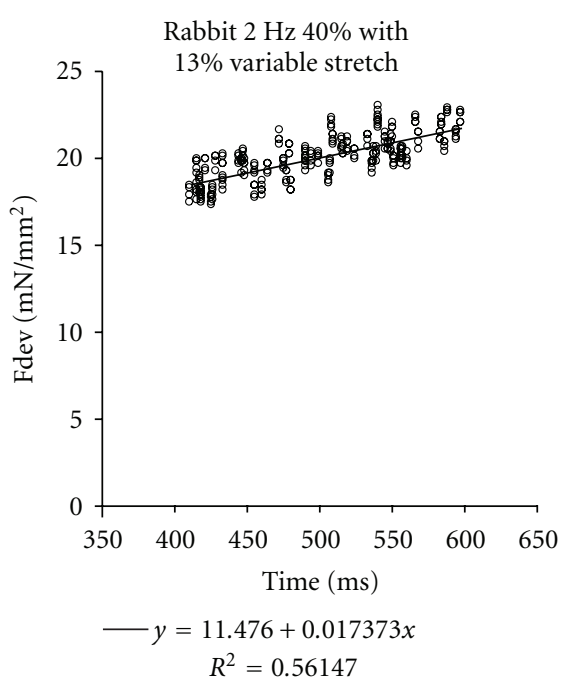

(b)

Figure 5: Top half displays the interaction between prebeat duration and force development at $2 \mathrm{~Hz}$. The correlation coefficients are increasingly tighter as the preload stretch is increasingly applied. On the bottom half, we depict the same phenomena at the higher frequency $(4 \mathrm{~Hz})$. The correlation here is more evident and significantly different statistically $(P<0.01)$ from the initial nonstretch correlation value of 0.56 .

interval thus can have variable effects that are species dependent. Its underlying mechanisms require further in vivo experiments as well as potential investigation of the long-term effects on protein expression and posttranslational modifications that are known to occur as a result of length [28] of frequency changes $[11,29]$.

\section{Acknowledgments}

This study was supported by an American Heart Association Established Investigator Award 0740040N (to P. M. L. Janssen) and by NIH K02HL083957 (to P. M. L. Janssen). 
[4] P. M. L. Janssen, "Myocardial contraction-relaxation coupling," American Journal of Physiology, vol. 299, no. 6, pp. H1741-H1749, 2010.

[5] B. Pieske, M. Sütterlin, S. Schmidt-Schweda et al., "Diminished post-rest potentiation of contractile force in human dilated cardiomyopathy: functional evidence for alterations in intracellular Ca2+ handling," Journal of Clinical Investigation, vol. 98, no. 3, pp. 764-776, 1996.

[6] D. Burkhoff, D. T. Yue, M. R. Franz, W. C. Hunter, and K. Sagawa, "Mechanical restitution of isolated perfused canine left ventricles," American Journal of Physiology, vol. 246, no. 1, pp. H8-16, 1984.

[7] S. M. C. Hardman, M. I. M. Noble, T. Biggs, and W. A. Seed, "Evidence for an influence of mechanical restitution on beatto-beat variations in haemodynamics during chronic atrial fibrillation in patients," Cardiovascular Research, vol. 38, no. 1, pp. 82-90, 1998.

[8] F. L. Meijler, J. Strackee, J. L. Van Capelle, and J. C. Du Perron, "Computer analysis of the RR interval-contractility relationship during random stimulation of the isolated heart," Circulation Research, vol. 22, no. 5, pp. 695-702, 1968.

[9] C. A. A. Torres, K. D. Varian, and P. M. L. Janssen, "Variability in interbeat duration influences myocardial contractility in rat cardaic trabeculae," The Open Cardiovascular Medicine Journal, vol. 2, pp. 96-100, 2008.

[10] L. A. Mulieri, G. Hasenfuss, F. Ittleman, E. M. Blanchard, and N. R. Alpert, "Protection of human left ventricular myocardium from cutting injury with 2,3-butanedione monoxime," Circulation Research, vol. 65, no. 5, pp. 1441-1444, 1989.

[11] K. D. Varian and P. M. L. Janssen, "Frequency-dependent acceleration of relaxation involves decreased myofilament calcium sensitivity," American Journal of Physiology, vol. 292, no. 5, pp. H2212-H2219, 2007.

[12] G. E. Billman, Y. Nishijima, A. E. Belevych et al., "Effects of dietary omega-3 fatty acids on ventricular function in dogs with healed myocardial infarctions: in vivo and in vitro studies," American Journal of Physiology, vol. 298, no. 4, pp. H1219H1228, 2010.

[13] S. Raman, M. A. Kelley, and P. M. L. Janssen, "Effect of muscle dimensions on trabecular contractile performance under physiological conditions," Pflugers Archiv, vol. 451, no. 5, pp. 625-630, 2006.

[14] E. K. Rodriguez, W. C. Hunter, M. J. Royce, M. K. Leppo, A. S. Douglas, and H. F. Weisman, "A method to reconstruct myocardial sarcomere lengths and orientations at transmural sites in beating canine hearts," American Journal of Physiology, vol. 263, no. 1, pp. H293-H306, 1992.

[15] K. D. Varian, Y. Xu, C. A. A. Torres, M. M. Monasky, and P. M. L. Janssen, "A random cycle length approach for assessment of myocardial contraction in isolated rabbit myocardium," American Journal of Physiology, vol. 297, no. 5, pp. H1940H1948, 2009.

[16] Y. Xu, M. M. Monasky, N. Hiranandani, K. M. Haizlip, G. E. Billman, and P. M. Janssen, "Effect of Twitch Interval Duration on the Contractile Function of Subsequent Twitches in Isolated Rat, Rabbit, and Dog Myocardium under Physiological Conditions," Journal of Applied Physiology. In press.

[17] D. M. Bers, "Cardiac excitation-contraction coupling," Nature, vol. 415, no. 6868, pp. 198-205, 2002.

[18] M. M. Monasky and P. M. L. Janssen, "The positive force-frequency relationship is maintained in absence of sarcoplasmic reticulum function in rabbit, but not in rat myocardium," Journal of Comparative Physiology B, vol. 179, no. 4, pp. 469479, 2009.

[19] A. M. Lompre, J. J. Mercadier, C. Wisnewsky et al., "Speciesand age-dependent changes in the relative amounts of cardiac myosin isoenzymes in mammals," Developmental Biology, vol. 84, no. 2, pp. 286-290, 1981.

[20] A. T. M. Gosselink, P. K. Blanksma, H. J. G. M. Crijns et al., "Left ventricular beat-to-beat performance in atrial fibrillation: contribution of Frank-Starling mechanism after short rather than long RR intervals," Journal of the American College of Cardiology, vol. 26, no. 6, pp. 1516-1521, 1995.

[21] H. J. Muntinga, A. T. M. Gosselink, P. K. Blanksma, P. J. De Kam, E. E. Van Der Wall, and H. J. G. M. Crijns, "Left ventricular beat to beat performance in atrial fibrillation: dependence on contractility, preload, and afterload," Heart, vol. 82, no. 5, pp. 575-580, 1999.

[22] M. Petretta, M. L. E. Vicario, L. Spinelli et al., "Combined effect of the force-frequency and length-tension mechanisms on left ventricular function in patients with dilated cardiomyopathy," European Journal of Heart Failure, vol. 4, no. 6, pp. 727-735, 2002.

[23] C. Dumitrescu, P. Narayan, Y. Cheng, I. R. Efimov, and R. A. Altschuld, "Phase I and phase II of short-term mechanical restitution in perfused rat left ventricles," American Journal of Physiology, vol. 282, no. 4, pp. H1311-H1319, 2002.

[24] C. Luers, F. Fialka, A. Elgner et al., "Stretch-dependent modulation of $[\mathrm{Na}+] \mathrm{i},[\mathrm{Ca} 2+] \mathrm{i}$, and $\mathrm{pHi}$ in rabbit myocardium -a mechanism for the slow force response," Cardiovascular Research, vol. 68, no. 3, pp. 454-463, 2005.

[25] P. P. de Tombe, R. D. Mateja, K. Tachampa, Y. A. Mou, G. P. Farman, and T. C. Irving, "Myofilament length dependent activation," Journal of Molecular and Cellular Cardiology, vol. 48, no. 5, pp. 851-858, 2010.

[26] H. L. Granzier, Y. Wu, L. Siegfried, and M. LeWinter, “Titin: physiological function and role in cardiomyopathy and failure," Heart Failure Reviews, vol. 10, no. 3, pp. 211-223, 2005.

[27] M. W. Cooper, "Postextrasystolic potentiation: do we really know what it means and how to use it?" Circulation, vol. 88, no. 6, pp. 2962-2971, 1993.

[28] M. M. Monasky, B. J. Biesiadecki, and P. M. L. Janssen, "Increased phosphorylation of tropomyosin, troponin I, and myosin light chain-2 after stretch in rabbit ventricular myocardium under physiological conditions," Journal of Molecular and Cellular Cardiology, vol. 48, no. 5, pp. 10231028, 2010.

[29] R. R. Lamberts, N. Hamdani, T. W. Soekhoe et al., "Frequencydependent myofilament $\mathrm{Ca} 2+$ desensitization in failing rat myocardium," Journal of Physiology, vol. 582, no. 2, pp. 695709, 2007. 

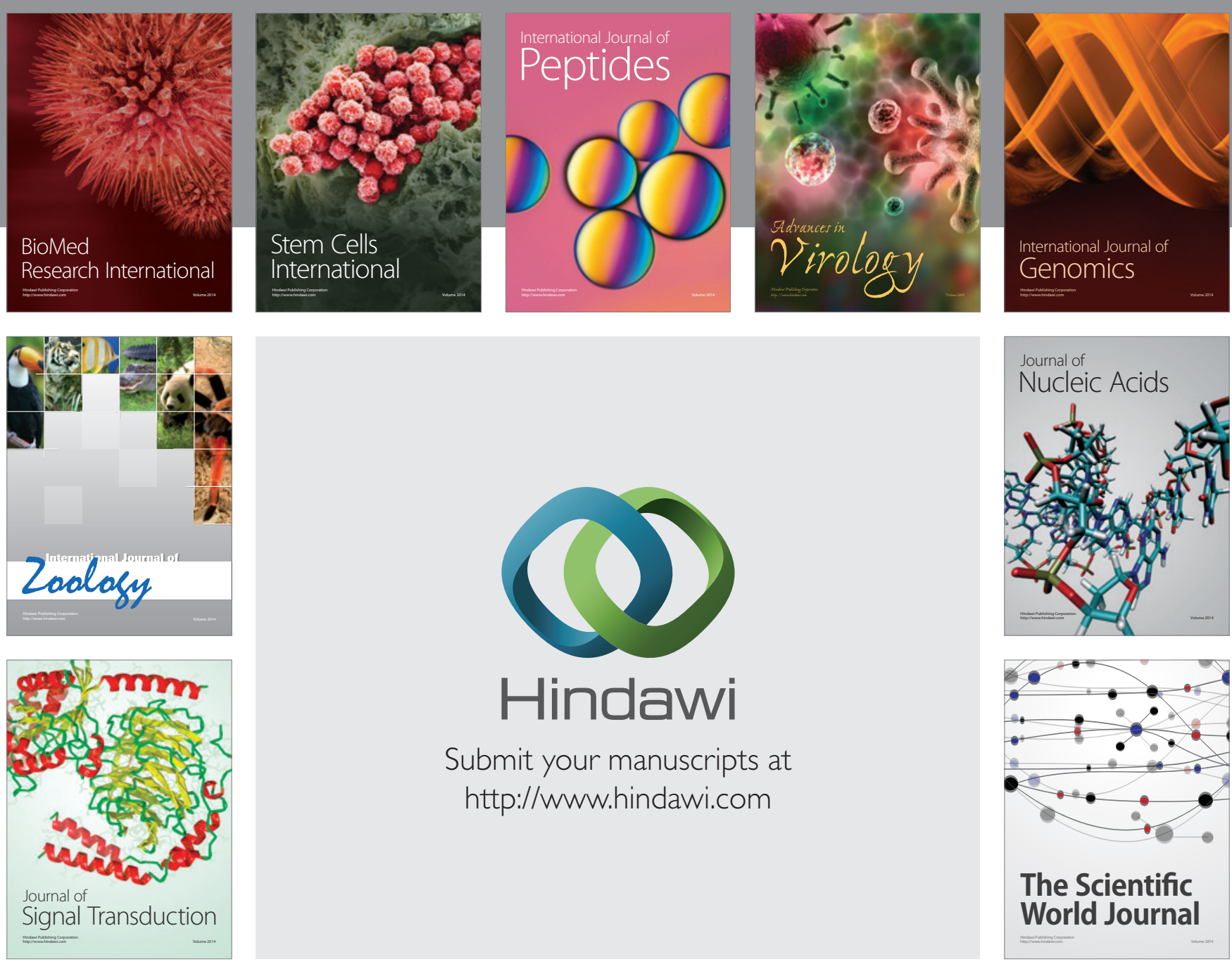

Submit your manuscripts at

http://www.hindawi.com
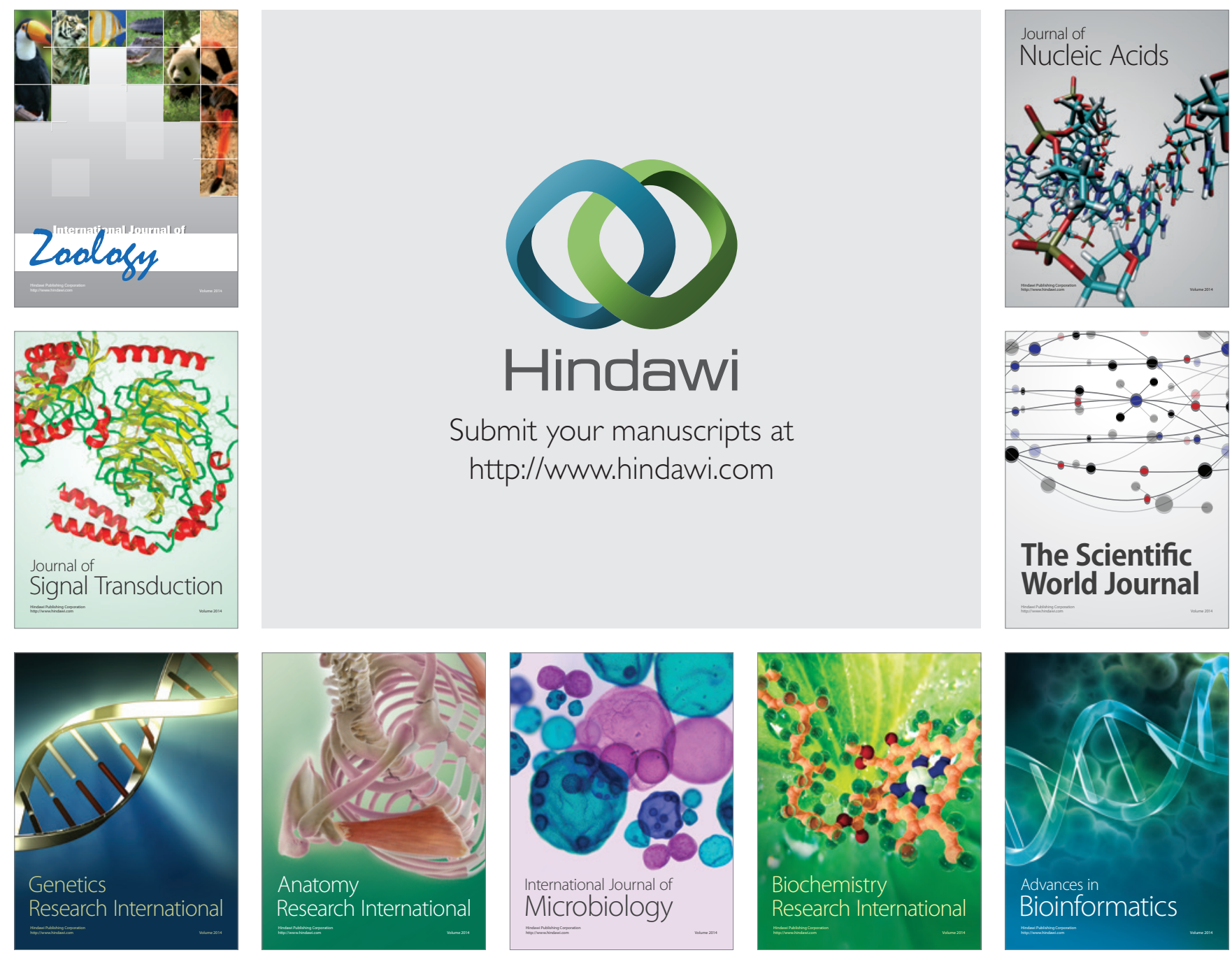

The Scientific World Journal
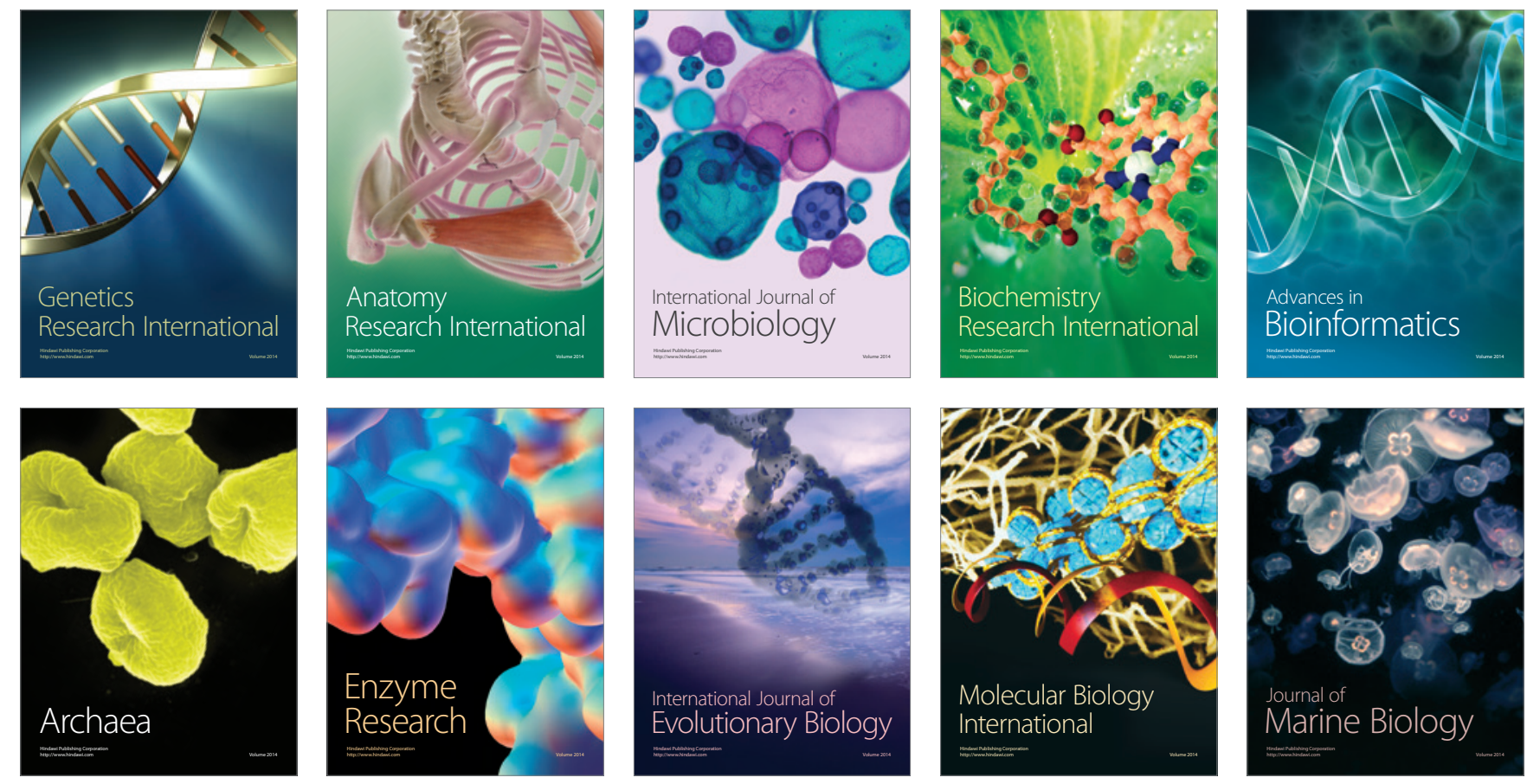